OPEN ACCESS

Edited by:

Giuseppe D'Antona,

University of Pavia, Italy

Reviewed by:

Tharmarajan Ramprasath, Georgia State University,

United States

Jie Qi,

Shanghai Normal University, China

*Correspondence:

Isabel Witvrouwen

Isabel.Witvrouwen@uantwerpen.be

Specialty section:

This article was submitted to

Exercise Physiology,

a section of the journal

Frontiers in Physiology

Received: 05 July 2021 Accepted: 02 September 2021 Published: 27 September 2021

Citation:

Witvrouwen I, Gevaert AB,

Possemiers N, Ectors B, Stoop T,

Goovaerts I, Boeren E, Hens W, Beckers PJ, Vorlat A, Heidbuchel $H$,

Van Craenenbroeck $A H$ and Van Craenenbroeck EM (2021)

Plasma-Derived microRNAs Are Influenced by Acute and Chronic Exercise in Patients With Heart Failure

With Reduced Ejection Fraction.

Front. Physiol. 12:736494. doi: 10.3389/fphys.2021.736494

\section{Plasma-Derived microRNAs Are Influenced by Acute and Chronic Exercise in Patients With Heart Failure With Reduced Ejection Fraction}

Isabel Witvrouwen ${ }^{1,2 *}$, Andreas B. Gevaert ${ }^{1,2}$, Nadine Possemiers ${ }^{2,3}$, Bert Ectors ${ }^{2}$, Tibor Stoop ${ }^{2}$, Inge Goovaerts'2, Evi Boeren', Wendy Hens ${ }^{2,3}$, Paul J. Beckers' 2,3 , Anne Vorlat ${ }^{1,2,3}$, Hein Heidbuchel ${ }^{1,2,3}$, Amaryllis H. Van Craenenbroeck ${ }^{4,5}$ and Emeline M. Van Craenenbroeck $k^{1,2,3}$

${ }^{1}$ Research Group Cardiovascular Diseases, GENCOR, University of Antwerp, Antwerp, Belgium, ${ }^{2}$ Department of Cardiology, Antwerp University Hospital, Edegem, Belgium, ${ }^{3}$ Cardiac Rehabilitation Centre, Antwerp University Hospital, Edegem, Belgium, ${ }^{4}$ Laboratory of Experimental Medicine and Paediatrics, University of Antwerp, Antwerp, Belgium, ${ }^{5}$ Department of Nephrology, University Hospitals Leuven, Leuven, Belgium

Background: Exercise training improves $\mathrm{VO}_{2}$ peak in heart failure with reduced ejection fraction (HFrEF), but the effect is highly variable as it is dependent on peripheral adaptations. We evaluated changes in plasma-derived miRNAs by acute and chronic exercise to investigate whether these can mechanistically be involved in the variability of exercise-induced adaptations.

Methods: Twenty-five male HFrEF patients (left ventricular ejection fraction $<40 \%$, New York Heart Association class $\geq$ II) participated in a 15-week combined strength and aerobic training program. The effect of training on plasma miRNA levels was compared to 21 male age-matched sedentary HFrEF controls. Additionally, the effect of a single acute exercise bout on plasma miRNA levels was assessed. Levels of 5 miRNAs involved in pathways relevant for exercise adaptation (miR-23a, miR-140, miR-146a, miR-191, and miR-210) were quantified using RT-qPCR and correlated with cardiopulmonary exercise test (CPET), echocardiographic, vascular function, and muscle strength variables.

Results: Expression levels of miR-146a decreased with training compared to controls. Acute exercise resulted in a decrease in miR-191 before, but not after training. Baseline miR-23a predicted change in $\mathrm{VO}_{2}$ peak independent of age and left ventricular ejection fraction (LVEF). Baseline miR-140 was independently correlated with change in load at the respiratory compensation point and change in body mass index, and baseline miR-146a with change in left ventricular mass index. 
Conclusion: Plasma-derived miRNAs may reflect the underlying mechanisms of exercise-induced adaptation. In HFrEF patients, baseline miR-23a predicted $\mathrm{VO}_{2}$ peak response to training. Several miRNAs were influenced by acute or repeated exercise. These findings warrant exploration in larger patient populations and further mechanistic in vitro studies on their molecular involvement.

Keywords: microRNA, HFrEF-heart failure with reduced ejection fraction, $\mathrm{VO}_{2}$ peak, peak oxygen uptake, response, exercise training, adaptation

\section{INTRODUCTION}

Heart failure (HF) is an increasingly prevalent syndrome with substantial mortality and morbidity due to exercise intolerance and dyspnea at exertion (Ponikowski et al., 2016). Apart from pharmacological treatment, exercise training is a successful multisystem approach in patients with heart failure with reduced ejection fraction (HFrEF) as it significantly improves morbidity and quality of life (Ponikowski et al., 2016). However, the individual response to exercise training in terms of peak oxygen consumption $\left(\mathrm{VO}_{2}\right.$ peak) is highly variable, with $55 \%$ of $\mathrm{HF}$ patients showing insufficient increase (Bakker et al., 2018). Importantly, these $\mathrm{VO}_{2}$ peak non-responders carry an adverse prognosis, independent of other risk factors, and early identification is mandatory (Tabet et al., 2008). The mechanisms driving the variability in response remain incompletely understood, but evidence is pointing toward both genetic and epigenetic regulation (Gevaert et al., 2019; Witvrouwen et al., 2019).

MicroRNAs (miRNAs) are epigenetic modulators of protein coding genes that act at the post-transcriptional level (Peschansky and Wahlestedt, 2014). They are involved in pathways that are relevant for adaptation to exercise, such as changes in skeletal muscle function and angiogenesis, reduction of inflammation and response to hypoxia (Wada et al., 2011; Hecksteden et al., 2016; Welten et al., 2016; Seo et al., 2017; An et al., 2018; Zheng et al., 2018). We recently identified 5 circulating miRNA (miR-23a, miR-140, miR-146a, miR-191, and miR-210), that predicted the training-induced change in $\mathrm{VO}_{2}$ peak in $\mathrm{HFrEF}$ patients. In a bio-informatics analysis of their gene targets, this miRNA panel showed intriguing relations with biological pathways that could be involved in cardiovascular adaptation to exercise, such as vascular endothelial growth factor (VEGF) and mitogen-associated protein kinase (Witvrouwen et al., 2021). Furthermore, these miRNAs have been related to endothelial function and angiogenesis, skeletal muscle mass and function, and inflammatory processes, all relevant to exercise adaptation (Wada et al., 2011; Zhou et al., 2011; Zhu et al., 2016; Seo et al., 2017; Sun et al., 2017; Mitchell et al., 2018; Zheng et al., 2018; Du et al., 2019; Liu et al., 2019; Qiao et al., 2020).

Abbreviations: Aix, Augmentation index; AIx75, Heart rate corrected AIx; CBC, Complete blood count; CRT, Cardiac resynchronization therapy; ET, Exercise training; FMD, Flow mediated dilation; ICD, Implantable cardioverter defibrillator; IVS, Interventricular septum; IVSd, Interventricular septal end diastole; LAVi, Left atrial volume index; LVEDV, Left ventricular end diastolic volume; LVEF, Left ventricular ejection fraction; LVMi, Left ventricular mass index; PWV, Carotid-femoral pulse wave velocity; RCP, Respiratory compensation point; RER, Respiratory exchange ratio; UC, Usual care.
Previously, it has been shown that miR-146a levels at peak exercise are positively related with $\mathrm{VO}_{2} \max$, and miR-210 was negatively related to $\mathrm{VO}_{2}$ max in healthy subjects (Baggish et al., 2011; Bye et al., 2013). Both miR-146a and miR-210 have also been associated with the diagnosis of HF (Vegter et al., 2016). Furthermore, circulating miRNA levels are dynamically regulated by acute and chronic exercise. In healthy subjects, some miRNAs are down- or upregulated immediately after an acute exercise bout, and return to resting levels $24 \mathrm{~h}$ after an extended-duration acute exercise bout, depending on the tissues of origin or targets affected by exercise (Baggish et al., 2011, 2014; Nielsen et al., 2014). However, whether circulating miRNAs in HFrEF patients are dynamically regulated after a period of exercise training or by an acute exercise bout is currently unknown.

In this prospective cohort study, we aimed to evaluate whether plasma levels of miR-23a, miR-140, miR-146a, miR-191, and miR210 are influenced by a 15 -week exercise training program. In addition, we assessed the effect of an acute exercise bout on plasma miRNA levels, both in the untrained and trained status.

\section{MATERIALS AND METHODS}

\section{Patients and Study Design}

In this prospective cohort study, consecutive HFrEF patients that were referred for a 15-week supervised combined strength and moderate-intensity aerobic training program to the Cardiac Rehabilitation Centre of the Antwerp University Hospital (ET group) were compared to age-matched HFrEF patients receiving usual care without exercise training (UC group). Randomization into a training and non-training group was considered as nonethical in view of the strong indication for exercise training in HFrEF (Class IA indication) (Ponikowski et al., 2016). Patients were included when they completed at least 30 of the 45 sessions. The study complied with the Declaration of Helsinki and was approved by the ethics committee of the Antwerp University Hospital. Written informed consent was obtained from all participants.

The change in miRNA levels after a 15 -week exercise training program was investigated in the ET group and compared the UC group, and baseline miRNA levels were related to the change in $\mathrm{VO}_{2}$ peak. In the ET group only, the relation between baseline miRNA levels and change in cardiopulmonary exercise test (CPET), cardiac and vascular adaptation, and muscle strength was studied, and the effect of an acute exercise bout on the miRNA panel was assessed (Figure 1). 


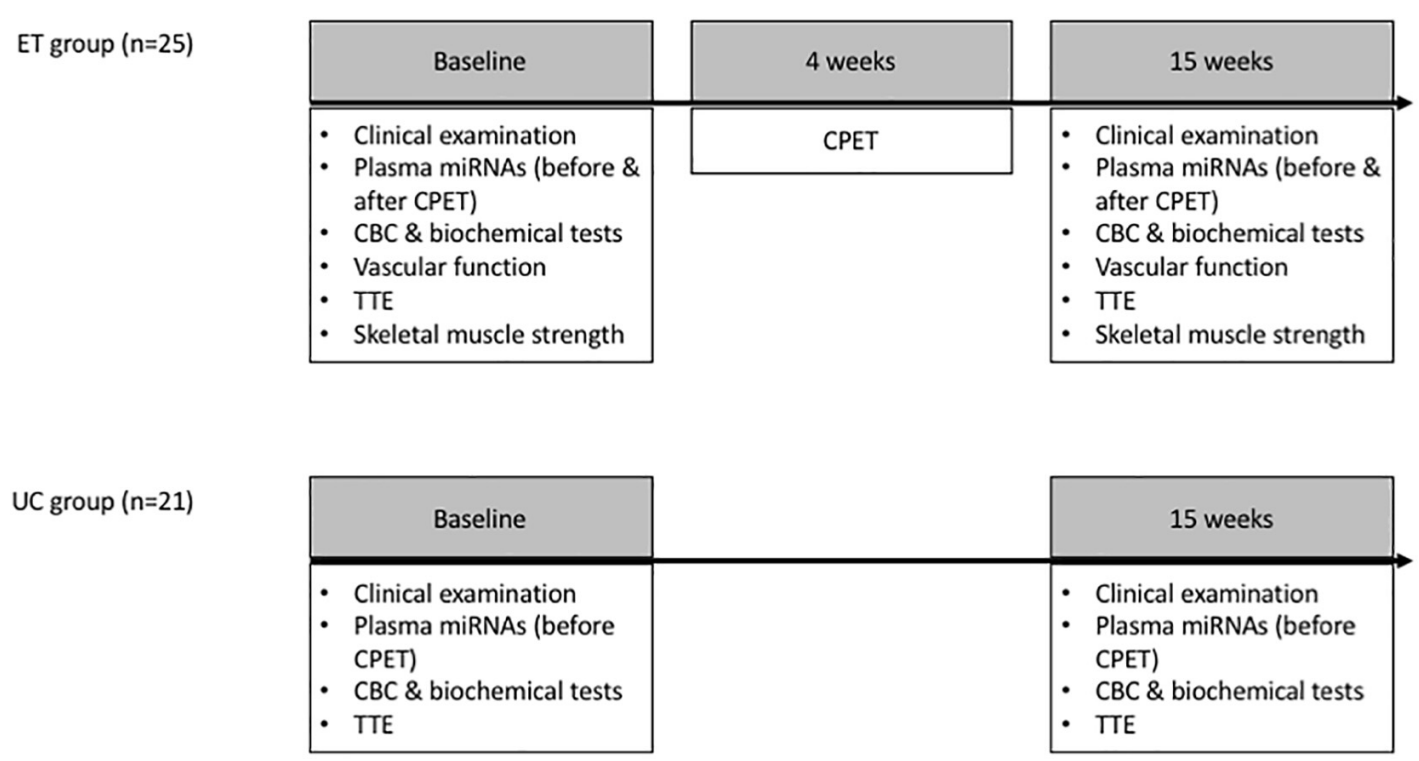

FIGURE 1 | Study design: Plasma miRNA levels were assessed at baseline and after 15 weeks in the ET and UC group. Vascular function, strength characteristics and the effect of an acute exercise bout (CPET) on the miRNA levels were evaluated in the ET group only. Since the relation between miRNAs and the central/peripheral determinants of $\mathrm{VO}_{2}$ peak (e.g., endothelial function and skeletal muscle strength) was not the primary objective of this study, these determinants were not assessed in the UC group. Vascular function measurements included flow mediated dilation of the brachial artery, pulse wave velocity and heart rate corrected augmentation index. Maximal strength of quadriceps, pectoral, latissimus dorsi, triceps, and deltoid muscles was assessed. CBC, complete blood count; CPET, cardiopulmonary exercise test; ET, exercise training; TTE, transthoracic echocardiography; UC, usual care.

\section{Power Calculation}

The sample size was calculated at 20 individuals per group. This offers $80 \%$ power to detect a difference in change in $\mathrm{VO}_{2}$ peak between the 2 groups of 0.9 standard deviations (SD) at a significance level of $5 \%$. Previous studies indicate that the standard deviation of the change in $\mathrm{VO}_{2}$ peak is typically around $1.4 \mathrm{ml} / \mathrm{kg} / \mathrm{min}$ (Belardinelli et al., 1995). Hence, a difference of $1.26 \mathrm{ml} / \mathrm{kg} / \mathrm{min}$ in change in $\mathrm{VO}_{2}$ peak between the two groups is detectable.

\section{In- and Exclusion Criteria}

Patients with a left ventricular ejection fraction (LVEF) $<40 \%$, symptoms and signs of HF [New York Heart Association class (NYHA) $\geq$ II], clinically stable and optimally medically treated for $\geq 6$ weeks, aged $\geq 18$ and $\leq 80$ years were eligible. To avoid the effect of sex-differences in epigenetic regulation, only male patients were included. Exclusion criteria were severe valvular pathology, severe renal failure (eGFR CKD$\mathrm{EPI}<30 \mathrm{ml} / \mathrm{min} / 1.73 \mathrm{~m}^{2}$ ), acute coronary syndrome $<4$ weeks ago, uncontrolled hypertension or arrhythmias, cognitive impairment, severe pulmonary disease (FEV $1<60 \%$ predicted, severe decrease in diffusion capacity, chronic obstructive pulmonary disease GOLD III-IV), auto-immune disorders, oncologic disease, or inability to exercise.

\section{Exercise Training}

Supervised in-hospital exercise training consisted of combined aerobic and resistance training, 3 sessions/week (58 min/session) for 15 weeks. Aerobic training intensity was set at $90 \%$ of heart rate (HR) at the respiratory compensation point (RCP). When RCP was not reached, exercise intensity was calculated using the Karvonen formula [exercise heart rate = rest heart rate $+\left(0.70^{*}\right.$ heart rate reserve $\left.)\right]$ (Karvonen et al., 1957). Strength exercise was an important component of the training program, with the focus primarily on gaining strength during the first 8 weeks. Afterward, aerobic training became more prominent (Supplementary Figure 1).

\section{Clinical Assessments}

CPET was performed on a treadmill (Medical Jaeger, Würzburg, Germany) with a graded protocol (equivalents of $40 \mathrm{~W}+20$ $\mathrm{W} / \mathrm{min}$ or $20 \mathrm{~W}+10 \mathrm{~W} / \mathrm{min}$ ) (Beckers et al., 2011), with an identical protocol for the follow-up test (Cardiovit CS200 Ergo-Spiro, Schiller AG, Baar, Switzerland). Gas exchange measurements and 12-lead electrocardiogram were recorded continuously. Blood pressure was measured every minute. $\mathrm{VO}_{2}$ peak was determined as the mean $\mathrm{VO}_{2}$ peak during the final $30 \mathrm{~s}$ of exercise. Percent predicted $\mathrm{VO}_{2}$ peak was calculated using the Jones equation (Jones and Campbell, 1982). The RCP was estimated from the systematic increased ventilatory equivalent for $\mathrm{VCO}_{2}\left(\mathrm{VE} / \mathrm{VCO}_{2}\right)$ and the systematic decrease in end tidal partial pressure of $\mathrm{CO}_{2}\left(\mathrm{PETCO}_{2}\right)$ (Whipp et al., 1989; Algul et al., 2017).

Echocardiography was performed on a Vivid E95 cardiac ultrasound using the $4 \mathrm{~V}$ transducer for 3-D imaging and analyzed on Tomtec Arena). Left ventricular ejection fraction (LVEF), left ventricular mass index (LVMi), left ventricular end diastolic volume (LVEDV), left atrial volume index (LAVi), 
interventricular septum (IVS) thickness and diastolic parameters (E/A, E/e') were recorded. In the UC group, LVEF was calculated using Simpson's monoplane (4 chamber view) method on M5S transducer, AGFA IMPAX Agility 8.1.2, Vivid E95.

Endothelial-dependent vasodilation of the brachial artery was evaluated by flow mediated dilation (FMD) as previously described (ProSound alfa6, Hitachi-Aloka Medical Ltd.) (Van Craenenbroeck et al., 2015c; Mannaerts et al., 2019). FMD was expressed as the percent change in peak vessel diameter from the baseline value [(peak diameter - baseline diameter)/baseline diameter]. Endothelial-independent dilation was calculated accordingly after sublingual administration of nitroglycerine. Arterial stiffness was assessed with carotid-femoral pulse wave velocity (PWV) and pulse wave analysis (PWA) that calculates augmentation index (AIx) and heart rate corrected AIx (AIx75) using SphygmoCor (Atcor Medical), as previously described (Van Craenenbroeck et al., 2015b). All measurements were done in triplicate.

Bioelectrical impedance analysis was performed on an Omron BF306 Body Fat Monitor (Omron Healthcare Co., Ltd., Kyoto) using 2 electrodes (1 handle in each hand) to provide estimates of total lean mass and fat mass.

\section{Plasma MicroRNA Levels}

Whole blood was collected after an overnight fast prior to the CPET in ethylenediaminetetraacetic acid tubes (EDTA). The first $3 \mathrm{ml}$ of blood was discarded to prevent contamination with skin epithelial cells and endothelial cells. Samples were centrifuged within $30 \mathrm{~min}$ after collection $(1,500 \mathrm{~g}, 15 \mathrm{~min})$ at room temperature, and plasma was stored at $-80^{\circ} \mathrm{C}$.

MiR-23a, miR-140, miR-146a, miR-191, and miR-210 were quantified in plasma samples using miRNA RT-qPCR. In brief, plasma samples were thawed on ice and centrifuged for $10 \mathrm{~min}$ $\left(4^{\circ} \mathrm{C}, 16,000 \mathrm{~g}\right)$. RNA enriched for small RNAs (including miRNAs) was isolated using the mirVana Paris Kit (Thermo Fisher Scientific). Four hundred microliter 2X Denaturing Solution was added to $400 \mu \mathrm{l}$ of plasma. RNA was extracted using acid-phenol:chloroform and ethanol. The aliquoted eluate was immediately stored at $-20^{\circ} \mathrm{C}$. Reverse transcription and preamplification were performed using TaqMan miRNA primers (Thermo Fisher Scientific) and multiplex qPCR was done in a CFX96 thermal cycler (BioRad) as previously described (Van Craenenbroeck et al., 2015a). Raw Cq values were calculated in BioRad CFX manager software v.3.1 using automatic baseline and threshold settings. Cq values that were undetermined or $>35$ were removed from the analysis, to minimize statistical confounding by high quantification cycle values. Data were normalized using geNorm and relative miRNA levels were expressed as $\log \left(2^{-\Delta C q *} 10^{4}\right)$ (Gevaert et al., 2018).

\section{Statistical Data Analysis}

Data were analyzed using SPSS 26.0 and R version 3.6.0.

Normality of continuous variables was evaluated using Shapiro-Wilk test. Normally distributed data are expressed as mean \pm standard deviation (SD), skewed variables as median and range (1st-3rd quartile). Fisher-exact test was used for comparison of categorical variables, independent samples $T$-test or Mann-Whitney $U$-test for comparison of continuous variables. To assess changes with 15 weeks of ET or with acute exercise, linear mixed models were fitted using time and group or visit as fixed effects and patient ID as random effect, or paired samples $T$-test was used as appropriate.

Correlations were assessed using Pearson correlation analysis. Multiple linear regression analyses adjusting for age and baseline LVEF were performed to assess independent determinants of $\mathrm{VO}_{2}$ peak. A two-sided $p$-value $<0.05$ was considered significant.

\section{RESULTS}

\section{Baseline Patient Characteristics and MicroRNA Expression}

Twenty-five patients were included in the ET group and 21 patients in the UC group. Baseline patient demographics, clinical, pharmacological, CPET characteristics, and circulating miRNA levels are shown in Table $\mathbf{1 .}$

At baseline, ET and UC were similar with regard to demographics and clinical characteristics, except for BMI, which was higher in UC $(p=0.042)$. Ischemic cardiomyopathy was more common in ET compared to UC $(p=0.033)$, and implantable cardioverter defibrillator (ICD) was less common in ET compared to UC $(p=0.022)$. Pharmacological therapy was comparable between ET and UC. CPET characteristics were similar between groups, except for work economy, which was lower in ET compared to UC group (6.4 vs. 7.3, $p=0.015$ ).

Baseline miRNA expression was similar between groups, except for miR-23a which was higher in patients referred for ET compared to CG $(p=0.043)$.

At baseline, better heart (LVEF) and kidney (creatinine) function were associated with higher $\mathrm{VO}_{2}$ peak (respectively, $r=0.303, p=0.043$, and $r=-0.514, p<0.001$, Supplementary Figure 2). Patients with lower LVEF had higher miR-210 levels $(r=-0.321, p=0.032$, Supplementary Figure 2) independent from age $(\beta=-9.455, p=0.035,95 \%$ C.I. $-18.192,-0.717)$. None of the other baseline miRNA levels were related with LVEF. No significant correlation was found between baseline miRNA levels and baseline $\mathrm{VO}_{2}$ peak.

\section{Exercise Training-Induced Changes in MicroRNA Expression}

Changes in aerobic capacity and clinical characteristics after 15 weeks of follow-up are shown in Table 2 and Supplementary Figure 3. Change in $\mathrm{VO}_{2}$ peak was significantly different between the ET and UC group ( +0.95 vs. $-0.64 \mathrm{ml} / \mathrm{kg} / \mathrm{min}$ (difference $1.59,95 \%$ CI $0.06,3.12, p=0.041)$. NYHA class, peak load and load at RCP significantly improved in ET. Both ET and UC patients performed a maximal exercise test, evidenced by a high respiratory exchange ratio (RER).

After 15 weeks of follow-up, plasma levels of miR-146a significantly decreased in the ET group, whereas in the UC group plasma levels remained unaltered ( $\mathrm{p}$ interaction $<0.05$, Figure 2 thick black lines). A significant different evolution in expression levels of miR-191 was observed in ET compared to UC (decrease 
TABLE 1 | Baseline patient characteristics and training adherence.

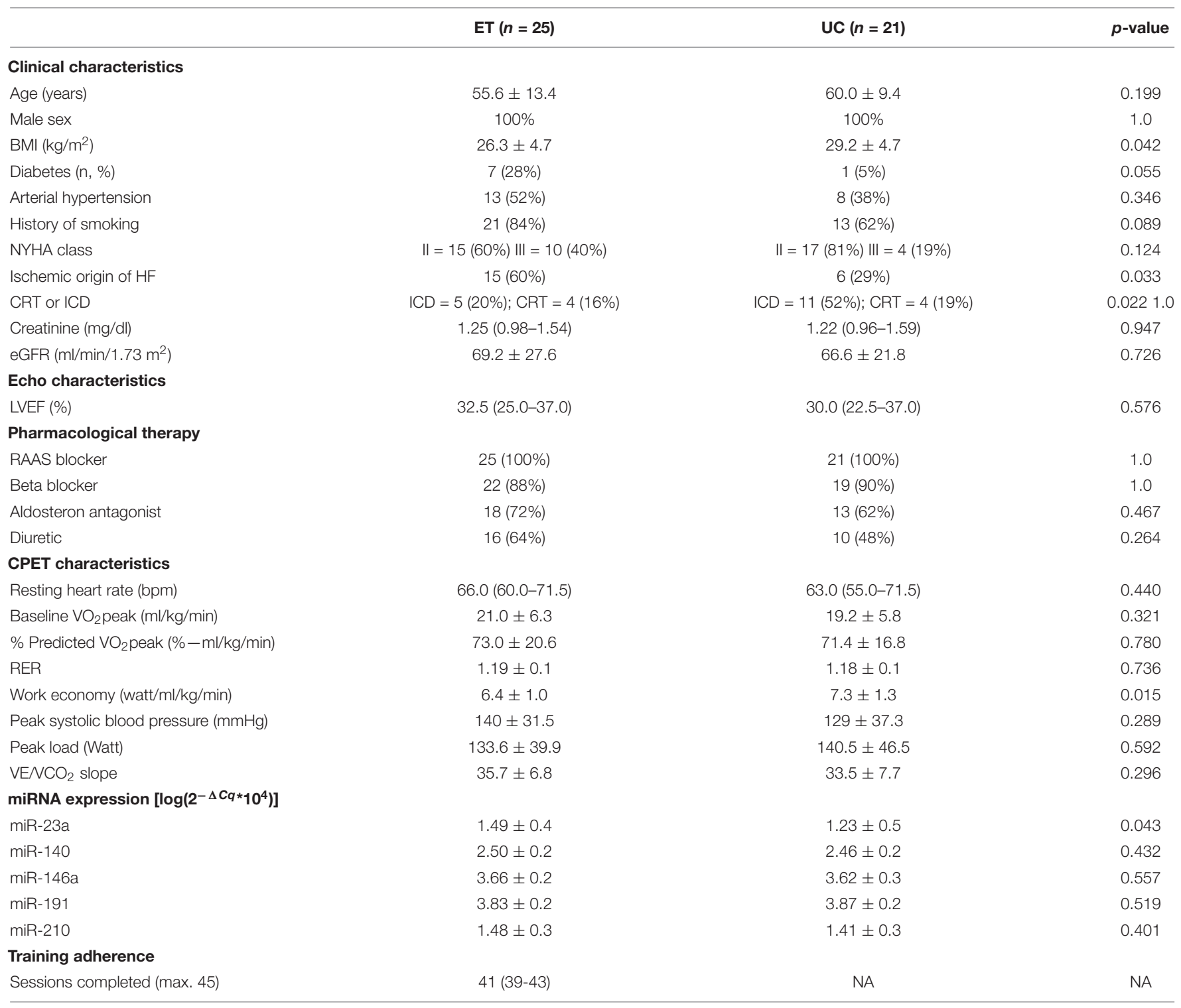

Data are expressed as mean $\pm S D$, as median (1st-3rd quartile) or as number of subjects (\%).

BMI, body mass index; ET, exercise training; CPET, cardiopulmonary exercise test; CRT, cardiac resynchronization therapy; eGFR, estimated glomerular filtration rate; ICD, implantable cardioverter defibrillator; HF, heart failure; LVEF, left ventricular ejection fraction; RAAS, renin-angiotensin-aldosterone system blockers; $n$, number of subjects; NA, not applicable; NYHA class, New York Heart Association functional class; RER, respiratory exchange ratio; UC, usual care.

vs. increase, $\mathrm{p}$ interaction $<0.05)$, but within group differences did not reach significance (dotted-dashed lines, Figure 2). None of the other miRNAs had a significant different evolution between the groups ( $\mathrm{p}$-interaction $>0.05$ ).

\section{Acute Exercise-Induced Changes in MicroRNA Expression}

A single exercise bout (CPET) resulted in a rapid and significant decrease in miR-191 levels in untrained HFrEF patients $(p=0.043)$. Intriguingly, exercise training resulted in a blunted and even reversed response to acute exercise (Figure 3); a non-significant increase $(p=0.120)$ after training was observed (p-interaction $=0.003)$. No significant effect on the other plasmaderived miRNAs was observed, but the same trend of reversal of the miRNA response was observed (except for miR-210).

\section{MicroRNAs as Predictors for Response to Exercise Training}

After 15 weeks of follow-up, $\mathrm{VO}_{2}$ peak significantly changed in ET compared to UC.

In the ET group only, changes in CPET, echocardiographic, muscle strength and vascular function parameters were assessed as secondary characteristics of adaptation to training. Following training, peak load, load at RCP, $\mathrm{VO}_{2}$ at $50 \%$ of peak load during 
CPET1, BMI, LVEF, LVMi, lean mass and strength characteristics significantly improved (see Table 2).

\section{Baseline MicroRNAs and Change in $\mathrm{VO}_{2}$ peak}

Baseline miR-23a was significantly associated with percent change in $\mathrm{VO}_{2}$ peak $(r=0.387, p=0.009$, Figure 4), and this was confirmed by multiple linear regression adjusted for age and baseline $\operatorname{LVEF}(\beta=11.307, p=0.017,95 \% \mathrm{CI}$ $2.113,20.500)$. Other miRNAs were not significantly related to $\mathrm{VO}_{2}$ peak changes.

\section{Baseline MicroRNAs and Training-Induced Changes in Clinical Variables}

Baseline miR-140 was related with the percent change in load at $\mathrm{RCP}(r=-0.505, p=0.033)$ as well as the percent change in BMI $(r=-0.454, p=0.023)$. Baseline miR-146a correlated with the percent change in LVMi $(r=-0.446, p=0.026$, Figure 5). None of the other baseline miRNAs were related with training-induced changes in clinical variables.

The percent change in BMI, percent change in peak load and percent change in lean mass were not related with the percent change in strength characteristics.

\section{DISCUSSION}

In this prospective cohort study, we investigated the effect of 15 weeks of exercise training as well as an acute exercise bout on plasma miRNA levels in HFrEF patients. Moreover, we studied the relation of miRNA levels with $\mathrm{VO}_{2}$ peak training response to unravel the underlying mechanisms of adaptation to chronic exercise. The principal findings include:

TABLE 2 | Change in clinical characteristics, CPET variables, echocardiographic findings, skeletal muscle strength, and vascular function after 15 weeks of either exercise training (ET) or usual care (UC).

\begin{tabular}{|c|c|c|c|c|c|}
\hline & \multicolumn{2}{|c|}{ ET $(n=25)$} & \multicolumn{2}{|c|}{ UC $(n=21)$} & \multirow[t]{2}{*}{$p$-value for interaction } \\
\hline & Baseline & 15 weeks & Baseline & 15 weeks & \\
\hline BMl & $26.3 \pm 4.7$ & $27.0 \pm 4.7^{\star}$ & $29.2 \pm 4.7$ & $29.1 \pm 4.6$ & 0.006 \\
\hline NYHA class (n, \%) & $\|=15(60 \%)\| I I=,10(40 \%)$ & $\begin{array}{c}I=9(36 \%), I I=13(52 \%) \\
I I I=2(8 \%), I V=1(4 \%)^{\star}\end{array}$ & $\begin{array}{c}\|=17(81 \%),\| \mid=4 \\
(19 \%)\end{array}$ & $\begin{array}{c}I=1(5 \%), I=14 \\
(67 \%), I I I=6(28 \%)\end{array}$ & 0.002 \\
\hline $\mathrm{VO}_{2}$ peak (ml/kg/min) & $21.0 \pm 6.3$ & $21.95 \pm 7.5$ & $19.2 \pm 5.8$ & $18.56 \pm 6.2$ & 0.041 \\
\hline Peak load (Watt) & $133.6 \pm 39.9$ & $156.4 \pm 47.9^{\star}$ & $140.5 \pm 46.5$ & $143.3 \pm 45.3$ & $<0.001$ \\
\hline RER & $1.19 \pm 0.1$ & $1.21 \pm 0.1$ & $1.18 \pm 0.1$ & $1.18 \pm 0.1$ & 0.675 \\
\hline VE $/ \mathrm{NCO}_{2}$ slope & $35.7 \pm 6.8$ & $37.2 \pm 9.4$ & $33.5 \pm 7.7$ & $35.5 \pm 9.5$ & 0.751 \\
\hline Load at RCP (Watt) & $110.5 \pm 38.5$ & $127.6 \pm 40.7^{\star \star}$ & $128.8 \pm 46.2$ & $113.8 \pm 52.6$ & 0.031 \\
\hline $\mathrm{VO}_{2}$ at $\mathrm{RCP}(\mathrm{ml} / \mathrm{kg} / \mathrm{min})$ & $19.2 \pm 6.1$ & $20.1 \pm 6.1$ & $18.5 \pm 6.7$ & $17.7 \pm 7.3$ & 0.370 \\
\hline $\begin{array}{l}\mathrm{VO}_{2} \text { at } 50 \% \text { of peak load } \\
\text { during CPET1 }(\mathrm{ml} / \mathrm{kg} / \mathrm{min})\end{array}$ & $14.1 \pm 4.1$ & $12.9 \pm 3.8^{\star \star}$ & $11.6 \pm 3.9$ & $12.0 \pm 4.7$ & 0.022 \\
\hline LVEF (\%) & $31.17 \pm 7.4$ & $37.15 \pm 9.9^{*}$ & & & \\
\hline LVMi (g/m2) & $161.32 \pm 72.0$ & $135.45 \pm 63.0^{\star *}$ & & & \\
\hline RWT & $0.33 \pm 0.09$ & $0.32 \pm 0.08$ & & & \\
\hline LAVi (ml/m2) & $45.36 \pm 19.3$ & $42.47 \pm 16.7$ & & & \\
\hline IVSd (mm) & $10.66 \pm 2.3$ & $10.55 \pm 2.0$ & & & \\
\hline LVEDV (ml) & $194.17 \pm 55.9$ & $193.58 \pm 54.8$ & & & \\
\hline $\mathrm{E} / \mathrm{A}$ & $1.29 \pm 0.8$ & $1.22 \pm 0.7$ & & & \\
\hline E/e' (med) & $17.4 \pm 8.2$ & $19.1 \pm 14.6$ & & & \\
\hline E/e' (lat) & $13.8 \pm 8.2$ & $12.7 \pm 9.1$ & & & \\
\hline Lean mass (kg) & $59.6 \pm 8.5$ & $61.5 \pm 8.1^{\star \star}$ & & & \\
\hline Bio-impedance (\%) & $26.0 \pm 7.2$ & $26.1 \pm 6.9$ & & & \\
\hline Quadriceps (kg) & $37.07 \pm 18.0$ & $51.20 \pm 19.2^{\star}$ & & & \\
\hline Latissimus dorsi (kg) & $46.25 \pm 12.3$ & $54.20 \pm 12.3^{*}$ & & & \\
\hline $\begin{array}{l}\text { Triceps, pectoral and } \\
\text { deltoid muscles (kg) }\end{array}$ & $55.80 \pm 14.3$ & $63.95 \pm 11.6^{\star \star}$ & & & \\
\hline Pectoral muscles (kg) & $28.88 \pm 10.9$ & $41.33 \pm 10.2^{*}$ & & & \\
\hline PWV (m/s) & $7.96 \pm 2.0$ & $7.63 \pm 1.9$ & & & \\
\hline FMD (\%) & $4.89 \pm 3.2$ & $5.18 \pm 2.3$ & & & \\
\hline Alx75 (\%) & $17.06 \pm 13.4$ & $17.5 \pm 13.0$ & & & \\
\hline
\end{tabular}

Data are expressed as mean $\pm S D$ or as number of subjects (\%). ${ }^{*} p<0.001,{ }^{* *} p<0.05$

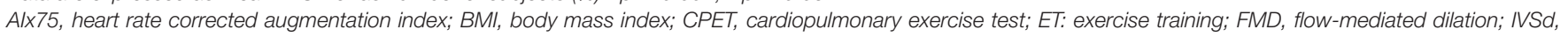

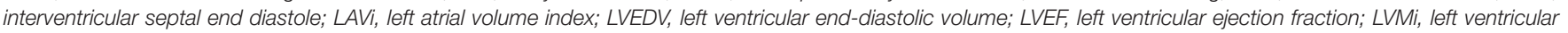

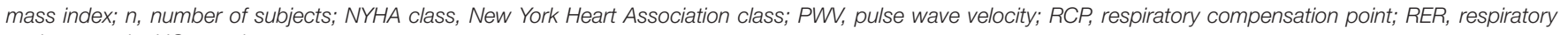
exchange ratio; UC, usual care. 
ET

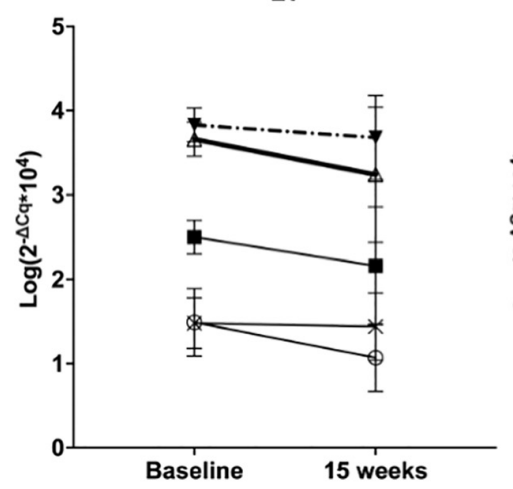

UC

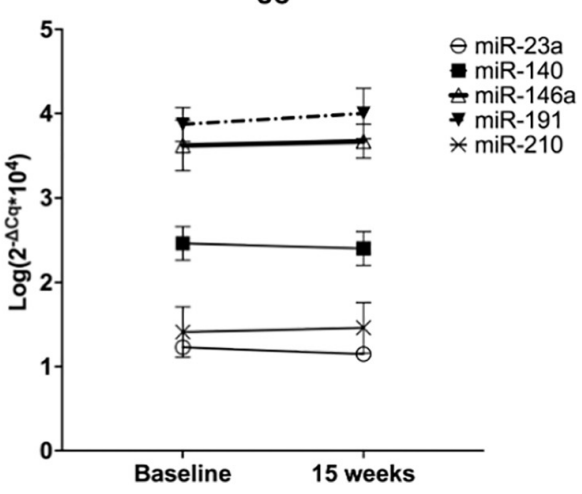

FIGURE 2 | Effect of 15 weeks of training on plasma levels of miRNAs in ET compared to 15 weeks of follow-up in UC. Data are expressed as the mean logarithm of the relative expression of the respective miRNA \pm SD at baseline and after 15 weeks. Each line represents the change in plasma miRNA levels with 15 weeks of training in ET $(n=25)$ and 15 weeks of follow-up in UC $(n=21)$.

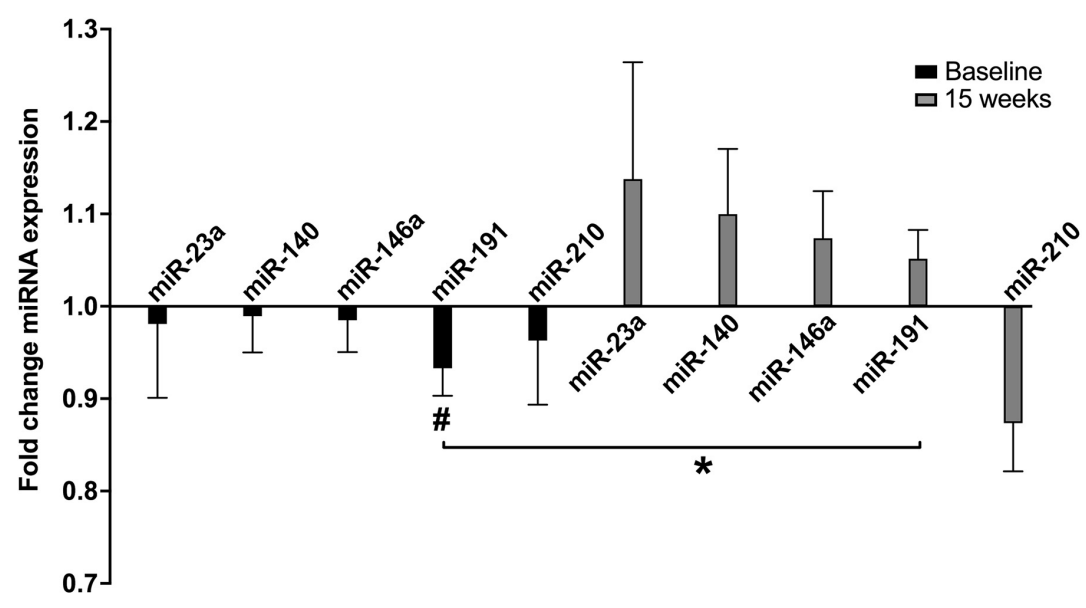

FIGURE 3 | Fold change miRNA expression with acute exercise at baseline and after 15 weeks of exercise training in ET. ET, exercise training group ( $n=25$ ); Fold change, post CPET/pre CPET miRNA expression. Data are expressed as mean and error. ${ }^{*}$ within group $p<0.05$, ${ }^{*} p$-value for interaction $<0.05$.

- miR-146a levels decrease following 15 weeks of training compared to controls

- A single bout of acute exercise results in a decrease in miR191 in untrained patients

- Baseline miR-23a predicts the percent change in $\mathrm{VO}_{2}$ peak following 15 weeks of training

- miRNA change in response to exercise may provide insights in the mechanisms driving VO2peak variability.

\section{Dynamic Regulation of MicroRNA Expression Following Chronic Exercise}

As previously reported, expression levels of circulating miRNAs change with acute or chronic exercise training (Baggish et al., 2011; Denham and Prestes, 2016). In the present study, we observed a significant decrease in relative expression of miR23a, miR-140, and miR-146a in the ET group with 15 weeks of training. However, the evolution was only significantly different for miR-146a when compared to the UC group. Our findings are in contrast with Baggish et al. (2011) who observed no change in miR-146a levels with 90 days of rowing training. This difference might be related to the population studied i.c. athletes. To date, evidence on the physiological role of circulating miRNA in the adaptation to exercise is scarce, and to the best of our knowledge, virtually non-existent in the response to training in HFrEF patients. Hence, we can only speculate that the differences in circulating miRNA levels after training that we observed, may result from an underlying active and selective miRNA process that is involved in pathways relevant to exercise adaptation in HFrEF patients, rather than reduced passive release of these miRNAs.

In HFrEF patients, capillary density in skeletal muscle is reduced (Duscha et al., 1999). Both miR-23a and miR-146a were previously shown to stimulate angiogenesis (Zhou et al., 2011; Zhu et al., 2016). Therefore, reduced miR-23a and miR146a levels after 15 weeks of training may reflect a diminished need for angiogenesis since capillary density increases with endurance and resistance training (Ingjer, 1979; Hudlicka et al., 


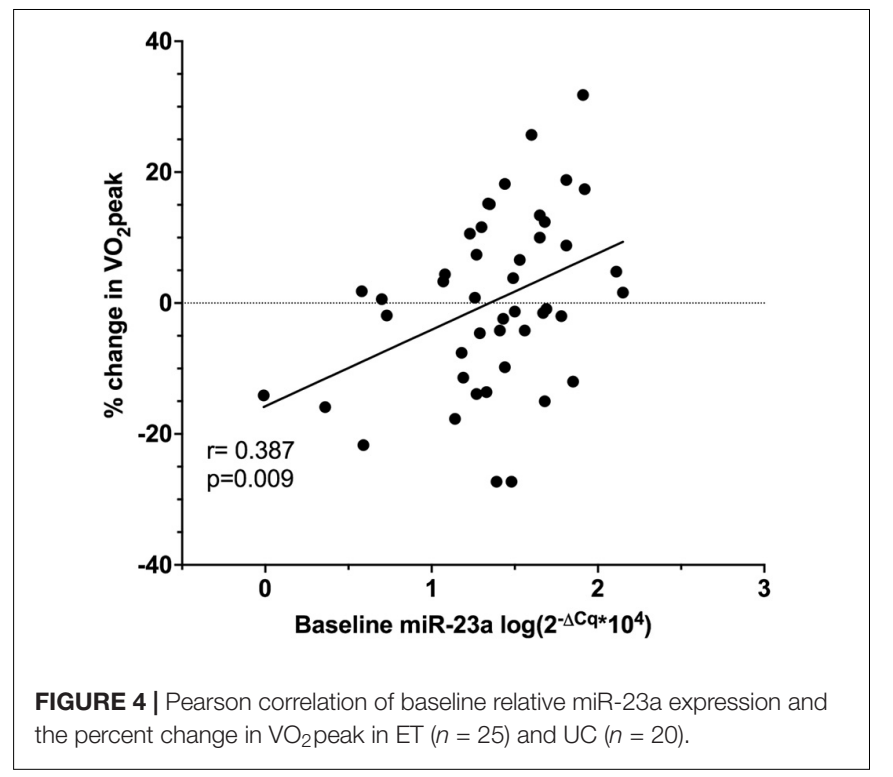

1992; Holloway et al., 2018). Also, a transient increase in miR23a and miR-146a may be expected during the training program, reflecting the exercise-induced angiogenesis, but this needs to be explored in future experiments.

Furthermore, HFrEF patients often have skeletal muscle wasting, especially with more advanced disease status, and this contributes to typical HF symptoms and signs such as dyspnea and exercise intolerance, which results in lower $\mathrm{VO}_{2}$ peak and load during CPET (Ponikowski et al., 2016). Exercise training improves skeletal muscle mass and function and has beneficial effects on LVEF and LV remodeling in HFrEF patients (Adams et al., 2017; Tucker et al., 2019). An important driver of skeletal muscle wasting is the ubiquitin-proteasome system (Adams et al., 2021). Both miR-23a and miR-140 were shown to protect against skeletal muscle atrophy through inhibiting the ubiquitinproteasome pathway and Wnt family member 11 expression, respectively (Wada et al., 2011; Liu et al., 2019). Hence, after training, sufficient skeletal muscle hypertrophy may result in lower miR-23a and miR-140 levels. However, this contrasts the finding that baseline miR-140 was inversely correlated with the change in load at RCP and BMI.

In the present study, we observed a differential expression between ET and UC in miR-23a. This could be attributed to the non-randomized study design, where ET patients might have had more skeletal muscle wasting compared to stable sedentary HFrEF controls, as BMI was significantly lower in ET compared to UC. Unfortunately, we do not have strength characteristics of the UC group. After 15 weeks of combined resistance and aerobic training, BMI significantly increased in the ET group, which could be attributed to increases in skeletal muscle mass, as indicated by higher strength characteristics in ER and coinciding increase in lean mass. However, no correlations with strength characteristics, or between (fold change) miR-23a and percent change in strength or lean mass were observed. Regarding the effect on cardiac hypertrophy, both miR-23a, miR-140, and miR-146a mediate cardiac hypertrophy through targeting the ubiquitin-proteasome pathway, GATA binding protein 4 and dihydrolipoyl succinyltransferase, respectively (Wang et al., 2012; Heggermont et al., 2017; Li et al., 2019). In contrast, we observed an inverse correlation between baseline miR-146a and percent change in LVMi in the ET group.

Baseline miR-210 was inversely related to LVEF. Since miR210 has been related to hypoxia and upregulates VEGF in endothelial cells (Zheng et al., 2018), the inverse relation with LVEF could reflect the reduced oxygen delivery to the periphery that coincides with worsening LVEF and cardiac output in HFrEF (Piepoli et al., 2010).

\section{Dynamic Regulation of MicroRNA Expression Following Acute Exercise}

In addition, miRNA levels can be altered by acute exercise bouts. Previous research in patients with chronic kidney disease showed a rapid downregulation of circulating miR-146a following an acute exercise bout (Van Craenenbroeck et al., 2015a). In patients with heart failure (average LVEF 47.7\%), Xu et al. (2016) observed an increase in circulating miR-21, miR-378, and miR-940 with acute exercise. However, in this study no distinction between heart failure with reduced, preserved or mid-range ejection fraction was made. In healthy athletes, miR-146a and miR-222 were shown to be upregulated by acute exercise both before
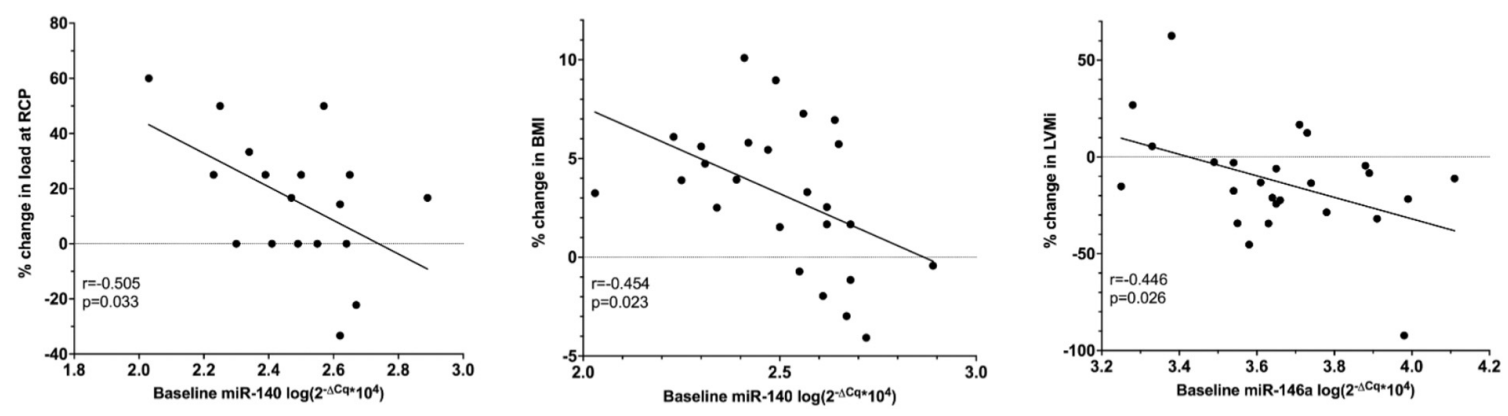

FIGURE 5 | Pearson correlation of baseline relative miRNA expression, the percent change in LVMi, percent change in load at RCP and the percent change in BMI in ET only. BMI, body mass index $(n=25)$; LVMi, left ventricular mass index $(n=25)$; RCP, respiratory compensation point $(n=18)$. 
and after a 90-day rowing training, whereas miR-21 and miR221 were only upregulated by acute exercise before the training period (Baggish et al., 2011). In the present study, at baseline all miRNA tended to decrease following an acute exercise bout, but this was only significant for miR-191. Intriguingly, this response reversed after 15 weeks of ET, which also suggests a selective training-induced effect on the miRNA expression.

MiR-191 has inhibitory effects on angiogenesis in endothelial cells (Gu et al., 2017; Du et al., 2019) and it stimulates myogenesis (Mitchell et al., 2018). As an acute exercise bout in sedentary patients elicits a hypoxic state, this triggers pro-angiogenic mechanisms. The fact that miR-191 has been shown to inhibit angiogenesis therefore could explain the decreased miR-191 levels observed at baseline. However, this needs to be confirmed in in vitro experiments. Regarding the effect on myogenesis, a single exercise bout provokes acute muscle damage after which myogenesis is established, and therefore lower levels of miR191. After this initial decrease in myogenesis, we speculate to observe a rise in miR-191 and stimulation of myogenesis to repair the damaged skeletal muscle cells and to increase skeletal muscle hypertrophy. However, we only collected blood samples immediately after CPET so this hypothesis needs to be confirmed. In addition, increased angiogenesis and reduced myogenesis due to lower circulating miR-191 levels following an acute exercise bout may be conflicting. This could be explained by the fact that miRNA are tissue and disease specific, and circulating miRNA levels not always reflect intracellular levels (Pigati et al., 2010).

Finally, we hypothesize that with repeated acute exercise bouts (i.e., the effect of a 15-week training program) in HFrEF patients, the triggers for angiogenesis and myogenesis might have faded out due to increased capillarity and skeletal muscle mass, resulting in the opposite change of miRNA expression levels.

\section{Predicting Change in Aerobic Capacity Based on Baseline Plasma MicroRNA Levels}

More than half of the HFrEF patients who participate in an ET program may not increase their $\mathrm{VO}_{2}$ peak (Bakker et al., 2018) and despite many efforts, a predictive biomarker for $\mathrm{VO}_{2}$ peak response to training is still lacking. In our previous study, we identified several miRNA that were upregulated in patients with an unfavorable $\mathrm{VO}_{2}$ peak response (Witvrouwen et al., 2021). Among these miRNAs, miR-23a, miR-140, miR146a, miR-191, and miR-210 were involved in pathways relevant for exercise adaptation processes. In the current study, we observed a significant change in $\mathrm{VO}_{2}$ peak in ET compared to UC; however, the increase within ET was not significant, which could be explained by the fact that BMI significantly increased in ET. Consequently, the observed change in $\mathrm{VO}_{2}$ peak in $\mathrm{ml} / \mathrm{kg} / \mathrm{min}$ is underestimated. Furthermore, we confirmed that baseline miR-23a predicts the change in $\mathrm{VO}_{2}$ peak with training, which may reflect the underlying mechanisms of exercise adaptation since miR-23a was shown to stimulate angiogenesis and to protect against skeletal muscle atrophy (Wada et al., 2011; Zhou et al., 2011). However, we observed clear improvements in muscle strength, but no correlations with miR23a. This could be attributed to the low sample size. Nevertheless, miRNAs could emerge as promising epigenetic biomarkers of training response.

\section{Limitations and Future Perspectives}

Whereas aerobic training is known to improve endothelial function in stable coronary artery disease and HFrEF patients (Van Craenenbroeck et al., 2010, 2015c), and both aerobic, resistance and combined aerobic/resistance training showed similar improvements in FMD in patients with hypertension or prehypertension (Pedralli et al., 2020), we did not observe significant improvements in vascular function with 15 weeks of ET. This could be attributed to this subgroup analysis lacking statistical power to draw definitive conclusions.

Furthermore, the study can be biased due to the nonrandomized design. However, as stated in the methods, randomizing patients to a training and control group would have been unethical in view of the class IA recommendation of ET in HFrEF patients with favorable effects on morbidity, mortality and quality of life (Ponikowski et al., 2016). As findings of this study are hypothesis generating, they should be validated in larger prospective trials and in in vitro experiments. Future pre-clinical studies could investigate and compare the expression levels in tissue (skeletal muscle, endothelial cells) to the observed changes in plasma levels. Hence, the contribution of miRNA to exercise adaptation processes can be examined, as either miRNA post-transcriptionally influence gene expression or they can be an exercise-induced epiphenomenon in these tissues (f.ex. exercise-induced skeletal muscle hypertrophy results in an increased release of miRNAs in the circulation). This will aid in further unraveling of the underlying mechanisms of response to acute and chronic exercise.

\section{CONCLUSION}

The effect of acute and chronic exercise on the expression levels of 5 circulating miRNAs involved in pathways relevant for exercise adaptation (miR-23a, miR-140, miR-146a, miR-191, and miR210) was investigated in HFrEF patients admitted to a 15 -week combined strength and aerobic training program and compared to the sedentary usual care group.

MiR-146a levels decreased following 15 weeks of training compared to the UC group. A single bout of acute exercise resulted in a decrease in miR-191 levels before, but not after training. Baseline miRNA-23a levels were related with the change in $\mathrm{VO}_{2}$ peak. Furthermore, baseline miR-140 was inversely related to the percent change in load at RCP and BMI, and baseline miR-146a was inversely related to the percent change in LVMi following 15 weeks of training.

Therefore, miR-23a, miR-140, miR-146a, and miR-191 may provide insights in skeletal muscle, cardiac hypertrophy and angiogenic response to exercise in HFrEF patients. These findings warrant further exploration in larger patient populations and in molecular biology set-ups. 


\section{DATA AVAILABILITY STATEMENT}

The raw data supporting the conclusions of this article are available from the corresponding author upon request, for non-commercial purposes, without breaching participant confidentiality.

\section{ETHICS STATEMENT}

The studies involving human participants were reviewed and approved by the Ethics Committee of the Antwerp University Hospital Drie Eikenstraat 655, 2650 Edegem, Belgium. The patients/participants provided their written informed consent to participate in this study.

\section{AUTHOR CONTRIBUTIONS}

IW, AG, AVC, and EVC: conceptualization and writing-original draft. IW, NP, BE, TS, IG, WH, and PB: data collection. IW and EB: formal analysis. AG, WH, PB, AV, HH, AVC, and EVC: supervision. All authors contributed to the article and approved the submitted version.

\section{FUNDING}

This work was supported by the Flanders Research Foundation (Predoctoral mandate to IW 1194918N, senior

\section{REFERENCES}

Adams, V., Reich, B., Uhlemann, M., and Niebauer, J. (2017). Molecular effects of exercise training in patients with cardiovascular disease: focus on skeletal muscle, endothelium, and myocardium. Am. J. Physiol. Hear. Circ. Physiol. 313, $\mathrm{H} 72-\mathrm{H} 88$.

Adams, V., Wunderlich, S., Mangner, N., Hommel, J., Esefeld, K., Gielen, S., et al. (2021). Ubiquitin-proteasome-system and enzymes of energy metabolism in skeletal muscle of patients with HFpEF and HFrEF. ESC Hear. Fail. 2021:5. doi: 10.1002/ehf2.13405

Algul, S., Ozcelik, O., and Yilmaz, B. (2017). Evaluation of relationship between aerobic fitness level and range of isocapnic buffering periods during incremental exercise test. Cell Mol. Biol. 63, 78-82. doi: 10.14715/cmb/2017. 63.3.15

An, R., Feng, J., Xi, C., Xu, J., and Sun, L. (2018). MiR-146a Attenuates SepsisInduced Myocardial Dysfunction by Suppressing IRAK1 and TRAF6 via Targeting ErbB4 Expression. Oxid. Med. Cell Longev 2018:57. doi: 10.1155/ 2018/7163057

Baggish, A. L., Hale, A., Weiner, R. B., Lewis, G. D., Systrom, D., Wang, F., et al. (2011). Dynamic regulation of circulating microRNA during acute exhaustive exercise and sustained aerobic exercise training. J. Physiol. 589, 3983-3994.

Baggish, A. L., Park, J., Min, P. K., Isaacs, S., Parker, B. A., Thompson, P. D., et al. (2014). Rapid upregulation and clearance of distinct circulating microRNAs after prolonged aerobic exercise. J. Appl. Physiol. 116, 522-531.

Bakker, E. A., Snoek, J. A., Meindersma, E. P., Hopman, M. T. E., Bellersen, L., Verbeek, A. L. M., et al. (2018). Absence of fitness improvement is associated with outcomes in heart failure patients. Med. Sci. Sport. Exerc. 50, 196-203. doi: 10.1249/MSS.00000000000 01429

Beckers, P. J., Possemiers, N. M., Van Craenenbroeck, E. M., Van Berendoncks, A. M., Wuyts, K., Vrints, C. J., et al. (2011). Impact of exercise testing mode on exercise parameters in patients with chronic heart clinical investigator grant to EVC 1804320N) and the King Baudouin Foundation.

\section{ACKNOWLEDGMENTS}

We would like to thank all the participants in this study and the staff of the Cardiac Rehabilitation Centre and Cardiology Department of the Antwerp University Hospital.

\section{SUPPLEMENTARY MATERIAL}

The Supplementary Material for this article can be found online at: https://www.frontiersin.org/articles/10.3389/fphys. 2021.736494/full\#supplementary-material

Supplementary Figure 1 | Training protocol including aerobic exercises (blue) and strength training (yellow). Latissimus dorsi, pectoral, triceps, deltoid, and quadriceps muscles were resistance trained. c-down, cool-down; ex, exercises; w-up, warming-up; reps, repetitions; R, respiratory compensation point at start; $\mathrm{RCPi}$, respiratory compensation point at 4w CPET; RM, repetition maximum.

Supplementary Figure 2 | Pearson correlation of baseline LVEF and creatinine with $\mathrm{VO}_{2}$ peak, and baseline LVEF and relative miR-210 expression in ET $(n=25)$ and UC group $(n=21)$. ET, exercise training; LVEF, left ventricular ejection fraction; UC, usual care.

Supplementary Figure 3 | Distribution of percent change in $\mathrm{VO}_{2}$ peak in all participants $(n=46)$. Gray, non-responders ( $<6 \%$ increase in $\mathrm{VO}_{2}$ peak); black, responders ( $\geq 6 \%$ increase in $\mathrm{VO}_{2}$ peak).

failure. Eur. J. Prev. Cardiol. 19, 389-395. doi: 10.1177/174182671140 0664

Belardinelli, R., Georgiou, D., Scocco, V., Barstow, T. J., and Purcaro, A. (1995). Low intensity exercise training in patients with chronic heart failure. J. Am. Coll. Cardiol. 26, 975-982.

Bye, A., Røsiø, H., Aspenes, S. T., Condorelli, G., Omland, T., and Wisloff, U. (2013). Circulating MicroRNAs and aerobic fitness The HUNT-Study. PLoS One 8:e57496. doi: 10.1371/journal.pone.00 57496

Denham, J., and Prestes, P. R. (2016). Muscle-Enriched MicroRNAs isolated from whole blood are regulated by exercise and are potential biomarkers of cardiorespiratory fitness. Front. Genet. 7:276-283. doi: 10.3389/fgene.2016. 00196

Du, K., Zhao, C., Wang, L., Wang, Y., Zhang, K.-Z., Shen, X.-Y., et al. (2019). MiR191 inhibit angiogenesis after acute ischemic stroke targeting VEZF1. Aging 11, $2762-2786$.

Duscha, B. D., Kraus, W. E., Keteyian, S. J., Sullivan, M. J., Green, H. J., Schachat, F. H., et al. (1999). Capillary density of skeletal muscle. a contributing mechanism for exercise intolerance in class II-III chronic heart failure independent of other peripheral alterations. JACC 33, 1956-1963.

Gevaert, A. B., Adams, V., Bahls, M., Bowen, T. S., Cornelissen, V., Dörr, M., et al. (2019). Towards a personalised approach in exercisebased cardiovascular rehabilitation: How can translational research help? A 'call to action' from the Section on Secondary Prevention and Cardiac Rehabilitation of the European Association of Preventive Cardiolo. Eur. J. Prev. Cardiol. 2019:16. doi: 10.1177/204748731987 7716

Gevaert, A. B., Witvrouwen, I., Vrints, C. J., Heidbuchel, H., Van Craenenbroeck, E. M., Van Laere, S. J., et al. (2018). MicroRNA profiling in plasma samples using qPCR arrays: Recommendations for correct analysis and interpretation. PLoS One 13:e0193173. doi: 10.1371/journal.pone.01 93173 
Gu, Y., Ampofo, E., Menger, M. D., and Laschke, M. W. (2017). MIR-191 suppresses angiogenesis by activation of NF$\mathrm{kB}$ signaling. FASEB J. 31, 3321-3333. doi: 10.1096/fj.201601 263R

Hecksteden, A., Leidinger, P., Backes, C., Rheinheimer, S., Pfeiffer, M., Ferrauti, A., et al. (2016). miRNAs and sports: Tracking training status and potentially confounding diagnoses. J. Transl. Med. 14, 1-13. doi: 10.1186/s12967-0160974-x

Heggermont, W. A., Papageorgiou, A.-P., Quaegebeur, A., Deckx, S., Carai, P., Verhesen, W., et al. (2017). Inhibition of MicroRNA-146a and overexpression of its target dihydrolipoyl succinyltransferase protect against pressure overload-induced cardiac hypertrophy and dysfunction. Circulation 136, 747-761. doi: 10.1161/CIRCULATIONAHA.116.02 4171

Holloway, T. M., Snijders, T., Van Kranenburg, J., Van Loon, L. J. C., and Verdijk, L. B. (2018). Temporal response of angiogenesis and hypertrophy to resistance training in young men. Med. Sci. Sports Exerc. 50, 36-45. doi: 10.1249/MSS. 0000000000001409

Hudlicka, O., Brown, M., and Egginton, S. (1992). Angiogenesis in skeletal and cardiac muscle. Physiol. Rev. 72, 369-417. doi: 10.1152/physrev.1992.72. 2.369

Ingjer, F. (1979). Effects of endurance training on muscle fibre ATP-ase activity, capillary supply and mitochondrial content in man. J. Physiol. 294, 419-432.

Jones, N., and Campbell, E. (1982). Clinical exercise testing. Saunders, Philadelphia

Karvonen, M. J., Kentala, E., and Mustala, O. (1957). The effects of training on heart rate; a longitudinal study. Ann. Med. Exp. Biol. Fenn. 35, 307-315.

Li, H., Xu, J.-D., Fang, X.-H., Zhu, J.-N., Yang, J., Pan, R., et al. (2019). Circular RNA circRNA_000203 aggravates cardiac hypertrophy via suppressing miR-26b-5p and miR-140-3p binding to Gata4. Cardiovasc. Res. 2019:215. doi: 10.1093/cvr/ cvz215

Liu, L., Li, T. M., Liu, X. R., Bai, Y. P., Li, J., Tang, N., et al. (2019). MicroRNA-140 inhibits skeletal muscle glycolysis and atrophy in endotoxin-induced sepsis in mice via the WNT signaling pathway. Am. J. Physiol. Cell Physiol. 317, C189-C199. doi: 10.1152/ajpcell.0041 9.2018

Mannaerts, D., Faes, E., Cornette, J., Gyselaers, W., Goovaerts, I., Roelant, E., et al. (2019). Low-flow mediated constriction as a marker of endothelial function in healthy pregnancy and preeclampsia: a pilot study. Pregnancy Hypertens. 17, 75-81. doi: 10.1016/j.preghy.2019. 02.001

Mitchell, C. J., D'Souza, R. F., Schierding, W., Zeng, N., Ramzan, F., O’Sullivan, J. M., et al. (2018). Identification of human skeletal muscle miRNA related to strength by high-throughput sequencing. Physiol. Genomics 50, 416-424.

Nielsen, S., Åkerström, T., Rinnov, A., Yfanti, C., Scheele, C., Pedersen, B. K., et al. (2014). The miRNA plasma signature in response to acute aerobic exercise and endurance training. PLoS One 9:e87308. doi: 10.1371/journal.pone.008 7308

Pedralli, M. L., Marschner, R. A., Kollet, D. P., Neto, S. G., Eibel, B., Tanaka, H., et al. (2020). Different exercise training modalities produce similar endothelial function improvements in individuals with prehypertension or hypertension: a randomized clinical trial Exercise, endothelium and blood pressure. Sci. Rep. 10, 1-9. doi: 10.1038/s41598-020-64365-x

Peschansky, V. J., and Wahlestedt, C. (2014). Non-coding RNAs as direct and indirect modulators of epigenetic regulation. Epigenetics 9, 3-12.

Piepoli, M. F., Guazzi, M., Boriani, G., Cicoira, M., Corrà, U., Libera, L. D., et al. (2010). Exercise intolerance in chronic heart failure: mechanisms and therapies. Part I. Eur. J. Prev. Cardiol. 17, 637-642. doi: 10.1097/HJR.0b013e32833 $61 \mathrm{dc} 5$

Pigati, L., Yaddanapudi, S. C. S., Iyengar, R., Kim, D. J., Hearn, S. A., Danforth, D., et al. (2010). Selective release of MicroRNA species from normal and malignant mammary epithelial cells. PLoS One 5:13515. doi: 10.1371/journal. pone.0013515

Ponikowski, P., Voors, A. A., Anker, S. D., Bueno, H., Cleland, J. G. F., Coats, A. J. S., et al. (2016). 2016 ESC Guidelines for the diagnosis and treatment of acute and chronic heart failure. Eur. Heart J. 37, 2129-2200. doi: 10.1093/ eurheartj/ehw128

Qiao, Y., Wang, C., Kou, J., Wang, I., Han, D., Huo, D., et al. (2020). MicroRNA23a suppresses the apoptosis of inflammatory macrophages and foam cells in atherogenesis by targeting HSP90. Gene 729:144319. doi: 10.1016/j.gene.2019. 144319

Seo, H.-H., Lee, S.-Y., Lee, C. Y., Kim, R., Kim, P., Oh, S., et al. (2017). Exogenous miRNA-146a Enhances the therapeutic efficacy of human mesenchymal stem cells by increasing vascular endothelial growth factor secretion in the ischemia/reperfusion-injured heart. J. Vasc. Res. 54, 100-108. doi: 10.1159/ 000461596

Sun, Y., Li, Y., Wang, H., Li, H., Liu, S., Chen, J., et al. (2017). MIR-146a$5 \mathrm{p}$ acts as a negative regulator of TGF- $\beta$ signaling in skeletal muscle after acute contusion. Acta Biochim. Biophys. Sin. 49, 628-634. doi: 10.1093/abbs/gm $\mathrm{x} 052$

Tabet, J. Y., Meurin, P., Beauvais, F., Weber, H., Renaud, N., Thabut, G., et al. (2008). Absence of exercise capacity improvement after exercise training program: a strong prognostic factor in patients with chronic heart failure. Circ. Heart Fail. 1, 220-226. doi: 10.1161/CIRCHEARTFAILURE.108.77 5460

Tucker, W. J., Beaudry, R. I., Liang, Y., Clark, A. M., Corey, R., Nelson, M. D., et al. (2019). Meta-analysis of exercise training on left ventricular ejection fraction in heart failure with reduced ejection fraction: a 10-year update. Prog. Cardiovasc. Dis. 62, 163-171. doi: 10.1016/j.pcad.2018.08.006.Meta-analysis

Van Craenenbroeck, A. H., Ledeganck, K. J., Van Ackeren, K., Jürgens, A., Hoymans, V. Y., Fransen, E., et al. (2015a). Plasma levels of microRNA in chronic kidney disease: patterns in acute and chronic exercise. Am. J. Physiol. Hear. Circ. Physiol. 309, 2008-2016.

Van Craenenbroeck, A. H., Van Craenenbroeck, E. M., Van Ackeren, K., Vrints, C. J., Conraads, V. M., Verpooten, G. A., et al. (2015b). Effect of moderate aerobic exercise training on endothelial function and arterial stiffness in CKD Stages 3-4: a randomized controlled trial. Am. J. Kidney Dis. 66, 285-296. doi: 10.1053/j.ajkd.2015.03.015

Van Craenenbroeck, E. M., Frederix, G., Pattyn, N., Beckers, P., Van Craenenbroeck, A. H., Gevaert, A., et al. (2015c). Effects of aerobic interval training and continuous training on cellular markers of endothelial integrity in coronary artery disease: A SAINTEX-CAD substudy. Am. J. Physiol. Hear. Circ. Physiol. 309, H1876-H1882. doi: 10.1152/ajpheart.00341. 2015

Van Craenenbroeck, E. M., Hoymans, V. Y., Beckers, P. J., Possemiers, N. M., Wuyts, K., Paelinck, B. P., et al. (2010). Exercise training improves function of circulating angiogenic cells in patients with chronic heart failure. Basic Res. Cardiol. 105, 665-676. doi: 10.1007/s00395-010-0 105-4

Vegter, E. L., van der Meer, P., de Windt, L. J., Pinto, Y. M., and Voors, A. A. (2016). MicroRNAs in heart failure: from biomarker to target for therapy. Eur. J. Heart Fail. 18, 457-468.

Wada, S., Kato, Y., Okutsu, M., Miyaki, S., Suzuki, K., Yan, Z., et al. (2011). Translational suppression of atrophic regulators by MicroRNA-23a integrates resistance to skeletal muscle atrophy. J. Biol. Chem. 286, 38456-38465. doi: 10.1074/jbc.M111.271270

Wang, K., Lin, Z. Q., Long, B., Li, J. H., Zhou, J., and Li, P. F. (2012). Cardiac hypertrophy is positively regulated by microRNA miR-23a. J. Biol. Chem. 287, 589-599. doi: 10.1074/jbc.M111.26 6940

Welten, S. M. J., Goossens, E. A. C., Quax, P. H. A., and Nossent, A. Y. (2016). The multifactorial nature of microRNAs in vascular remodelling. Cardiovasc. Res. 110, 6-22. doi: $10.1093 / \mathrm{cvr} / \mathrm{cv}$ w039

Whipp, B. J., Davis, J. A., and Wasserman, K. (1989). Ventilatory control of the "isocapnic buffering" region in rapidly-incremental exercise. Respir. Physiol. 76, 357-367. doi: 10.1016/0034-5687(89)90 076-5

Witvrouwen, I., Gevaert, A. B., Possemiers, N., Beckers, P. J., Vorlat, A., Heidbuchel, H., et al. (2021). Circulating microRNA as predictors for exercise response in heart failure with reduced ejection fraction. Eur. J. Prev. Cardiol. 2021:142. doi: 10.1093/eurjpc/zwa a142

Witvrouwen, I., Van Craenenbroeck, E. M., Abreu, A., Moholdt, T., and Kränkel, N. (2019). Exercise training in women with cardiovascular disease: differential response and barriers - review and perspective. Eur. J. Prev. Cardiol. 2019, 1-13. doi: $10.1177 / 2047487319838221$ 
Xu, T., Zhou, Q., Che, L., Das, S., Wang, L., Jiang, J., et al. (2016). Circulating miR21 , miR-378, and miR-940 increase in response to an acute exhaustive exercise in chronic heart failure patients. Oncotarget 7, 12414-12425. doi: 10.18632/ oncotarget.6966

Zheng, Z., Liu, L., Zhan, Y., Yu, S., and Kang, T. (2018). Adiposederived stem cell-derived microvesicle-released miR-210 promoted proliferation, migration and invasion of endothelial cells by regulating RUNX3. Cell Cycle 17, 1026-1033. doi: 10.1080/15384101.2018.148 0207

Zhou, Q., Gallagher, R., Ufret-Vincenty, R., Li, X., Olson, E. N., and Wang, S. (2011). Regulation of angiogenesis and choroidal neovascularization by members of microRNA-23 27 24 clusters. Proc. Natl. Acad. Sci. USA 108, 8287-8292. doi: 10.1073/pnas.110525 4108

Zhu, H. Y., Bai, W. D., Liu, J. Q., Zheng, Z., Guan, H., Zhou, Q., et al. (2016). Upregulation of FGFBP1 signaling contributes to MIR-146a-induced angiogenesis in human umbilical vein endothelial cells. Sci. Rep. 6, 1-11. doi: 10.1038/ srep 25272
Conflict of Interest: The authors declare that the research was conducted in the absence of any commercial or financial relationships that could be construed as a potential conflict of interest.

Publisher's Note: All claims expressed in this article are solely those of the authors and do not necessarily represent those of their affiliated organizations, or those of the publisher, the editors and the reviewers. Any product that may be evaluated in this article, or claim that may be made by its manufacturer, is not guaranteed or endorsed by the publisher.

Copyright (C) 2021 Witvrouwen, Gevaert, Possemiers, Ectors, Stoop, Goovaerts, Boeren, Hens, Beckers, Vorlat, Heidbuchel, Van Craenenbroeck and Van Craenenbroeck. This is an open-access article distributed under the terms of the Creative Commons Attribution License (CC BY). The use, distribution or reproduction in other forums is permitted, provided the original author(s) and the copyright owner(s) are credited and that the original publication in this journal is cited, in accordance with accepted academic practice. No use, distribution or reproduction is permitted which does not comply with these terms. 